\title{
Small scale response and modeling of periodically forced turbulence
}

\author{
Wouter J. T. Bos \\ LMFA, UMR CNRS 5509, Ecole Centrale de Lyon-Université Lyon 1-INSA de Lyon, 69134 Ecully, France \\ Timothy T. Clark \\ Tau Technologies, Albuquerque, New Mexico \\ Robert Rubinstein \\ NASA Langley Research Center, Hampton, Virginia
}

(Received 6 November 2006; accepted 15 March 2007; published online 10 May 2007)

\begin{abstract}
The response of the small scales of isotropic turbulence to periodic large scale forcing is studied using two-point closures. The frequency response of the turbulent kinetic energy and dissipation rate, and the phase shifts among production, energy, and dissipation are determined as functions of the Reynolds number. It is observed that the amplitude and phase of the dissipation exhibit nontrivial frequency and Reynolds number dependence that reveals a filtering effect of the energy cascade. Perturbation analysis is applied to understand this behavior which is shown to depend on distant interactions between widely separated scales of motion. Finally, the extent to which finite dimensional models (standard two-equation models and various generalizations) can reproduce the observed behavior is discussed. (C) 2007 American Institute of Physics. [DOI: 10.1063/1.2728939]
\end{abstract}

\section{INTRODUCTION}

Statistical transients in turbulence remain a major challenge to both theory and modeling. The mechanisms by which a turbulent flow readjusts to new conditions, for example in boundary layers with sudden changes in wall roughness or pressure gradient, ${ }^{1}$ are not entirely understood and continue to resist prediction by models.

Another class of statistically time-dependent turbulent flows is defined by the presence of periodically oscillating forcing. The classic example is steady pipe flow with small superposed oscillations of the mean pressure gradient. This flow has been the subject of extensive experimental, 2,3 theoretical, $^{4,5}$ and numerical ${ }^{6}$ investigation. There are two obvious limits: the "static" limit of slow oscillations, in which the turbulence evolves through a sequence of local steady states, and a limit of "frozen" turbulence in which the turbulence does not respond at all to the oscillations.

Analysis of oscillating pipe flow typically concentrates on the phase relations among the wall shear, centerline velocity, and pressure perturbation. These quantities prove remarkably difficult to predict at frequencies intermediate between the static and frozen limits even if the problem admits a linearized description, indicating unanticipated subtleties in the dynamics; indeed, the only entirely adequate predictions are by large-eddy simulation, ${ }^{6}$ which is very surprising in view of the apparent simplicity of the problem.

Recently, the problem of periodically forced homogeneous isotropic turbulence has been proposed ${ }^{7}$ and investigated theoretically, ${ }^{8}$ by numerical simulations, ${ }^{9}$ and by experiments using time-dependent grids. ${ }^{10}$ Because of the absence of complications such as near-wall behavior, this problem provides an ideal setting in which to investigate the time-dependent spectral dynamics of turbulence.

Previous work on this problem has been motivated by a search for resonance-like energy response near a critical fre- quency proportional to the inverse large-eddy turnover time, and perhaps at integer multiples of this frequency as well. This paper focuses instead on the properties of the dissipation rate. At frequencies intermediate between the static and frozen turbulence limits, nontrivial Reynolds numberdependent properties are found. The energy cascade acts like a low-pass filter that damps high-frequency oscillations, but as in the oscillating pipe flow, the details are more complex than the simple problem statement would suggest.

The main results are obtained by the eddy damped quasinormal Markovian (EDQNM) closure. ${ }^{11,12}$ The predictions of this closure for periodically forced turbulence are in reasonable qualitative agreement with existing results. Elementary arguments show that at forcing frequency $\omega$, the amplitude of the energy and dissipation rate oscillations vary as $\omega^{-1}$ for large frequencies. However, the calculations show that the dissipation rate modulation amplitude exhibits nontrivial $\omega^{-3}$ scaling in the intermediate frequency range, and the phase difference between the production and the dissipation rate has complex dependence on both $\omega$ and Reynolds number in this range.

To understand this behavior, we apply asymptotic analysis to two simpler models: the classical Heisenberg model ${ }^{13,14}$ and a recent generalization. ${ }^{15}$ In these models, the details of triad interactions are suppressed, but the essential idea of nonlocal interaction is retained. We show analytically how the energy cascade filters the oscillations, and that this filtering is responsible for the observations.

Some finite dimensional models of the two-equation type will be considered. The two-equation model is correct in both the static and frozen limits, but misses important features of the dynamics at intermediate frequencies, including the Reynolds number dependence of the dissipation. A more complex three-equation model allows for more complex phase relations, but is also incapable of capturing the Rey- 
nolds number dependence. It should be emphasized that this dependence is not a low Reynolds number effect.

A related problem involving periodic forcing is oscillatory homogeneous shear flow. ${ }^{16}$ This problem has important, and even dominant linear effects; it therefore has a somewhat different character from periodically forced isotropic turbulence, in which only nonlinear mechanisms are important. Another related problem can be mentioned, in which turbulence is forced periodically at the boundary of the flow region. ${ }^{17,18}$ This flow has many interesting similarities to periodically forced isotropic turbulence; although it is simpler in many respects than periodically modulated pipe flow, the dynamics of this problem may include effects of turbulent diffusion as well as energy transfer and may therefore not be entirely amenable to the present type of analysis.

\section{DEFINITIONS AND ELEMENTARY PROPERTIES}

The spectral evolution equation for time-dependent forced homogeneous isotropic turbulence is ${ }^{14}$

$$
\dot{E}(\kappa, t)=P(\kappa, t)-T(\kappa, t)-2 \nu \kappa^{2} E(\kappa, t),
$$

where $E(\kappa, t)$ is the energy spectrum and $T(\kappa, t)$ is the energy transfer due to nonlinear interactions. The production spectrum $P(\kappa, t)$ is assumed to be localized near some wavenumber $\kappa_{P}(t)$. Consider a basic steady state, defined by the timeindependent form of Eq. (1):

$$
0=\bar{P}(\kappa)-\bar{T}(\kappa)-2 \nu \kappa^{2} \bar{E}(\kappa) .
$$

The problem of periodically forced turbulence is formulated by introducing a periodic perturbation of the production spectrum,

$$
P(\kappa, t)=\bar{P}(\kappa)+\widetilde{P}(\kappa) \cos (\omega t),
$$

where we will assume the proportionality

$$
\widetilde{P}(\kappa)=c \bar{P}(\kappa)
$$

with $c \ll 1$, so that the problem can be analyzed by linearization about the steady state defined by Eq. (2). Then,

$$
E(\kappa, t)=\bar{E}(\kappa)+\delta E(\kappa, t)
$$

with $\delta E(\kappa, t) \ll \bar{E}(\kappa)$. If at sufficiently long times, $E(\kappa, t)$ becomes periodic in time, linearity implies that the period is $\omega$, hence,

$$
\delta E(\kappa, t)=\widetilde{E}(\kappa) \cos [\omega t+\psi(\kappa)] .
$$

In terms of the quantities

$$
\tilde{F}(\kappa)=\widetilde{E}(\kappa) \cos \psi(\kappa), \quad \widetilde{G}(\kappa)=\tilde{E}(\kappa) \sin \psi(\kappa),
$$

$\delta E(\kappa, t)$ is written as

$$
\delta E(\kappa, t)=\cos (\omega t) \tilde{F}(\kappa)-\sin (\omega t) \tilde{G}(\kappa) .
$$

The basic time-dependent single-point moments: total production $P(t)$, turbulent kinetic energy $k(t)$, and dissipation rate $\epsilon(t)$, are expressed in terms of their time averages $\bar{P}, \bar{k}$, and $\bar{\epsilon}$ and their phase averages $\widetilde{P}, \widetilde{k}$, and $\tilde{\epsilon}$ as

$$
\begin{aligned}
& P(t)=\bar{P}+\tilde{P} \cos (\omega t), \\
& k(t)=\bar{k}+\tilde{k} \cos \left(\omega t+\phi_{k}\right), \\
& \epsilon(t)=\bar{\epsilon}+\tilde{\epsilon} \cos \left(\omega t+\phi_{\epsilon}\right),
\end{aligned}
$$

where

$$
\begin{aligned}
& \bar{P}=\int_{0}^{\infty} d \kappa \bar{P}(\kappa), \quad \widetilde{P}=\int_{0}^{\infty} d \kappa \tilde{P}(\kappa), \\
& \bar{k}=\int_{0}^{\infty} d \kappa \bar{E}(\kappa), \quad \bar{\epsilon}=\int_{0}^{\infty} d \kappa 2 \nu \kappa^{2} \bar{E}(\kappa),
\end{aligned}
$$

and in view of Eq. (8),

$$
\begin{aligned}
& \tilde{k} \cos \left(\phi_{k}\right)=\int_{0}^{\infty} d \kappa \tilde{F}(\kappa), \quad \tilde{k} \sin \left(\phi_{k}\right)=\int_{0}^{\infty} d \kappa \tilde{G}(\kappa), \\
& \tilde{\epsilon} \cos \left(\phi_{\epsilon}\right)=\int_{0}^{\infty} d \kappa 2 \nu \kappa^{2} \widetilde{F}(\kappa), \\
& \tilde{\epsilon} \sin \left(\phi_{\epsilon}\right)=\int_{0}^{\infty} d \kappa 2 \nu \kappa^{2} \tilde{G}(\kappa) .
\end{aligned}
$$

For simplicity of notation, the spectral densities $\bar{P}(\kappa)$ and $\widetilde{P}(\kappa)$ are distinguished from the corresponding single-point moments $\bar{P}$ and $\widetilde{P}$ by their arguments rather than by a new letter.

The simplest formulation of the problem seeks the dependence of the phase averaged amplitudes $\widetilde{k}$ and $\widetilde{\boldsymbol{\epsilon}}$ and the phase shifts $\phi_{k}, \phi_{\epsilon}$ on the forcing frequency $\omega ; \tilde{k}$ will be called the modulated energy and $\tilde{\epsilon}$ the modulated dissipation. $P(t), k(t)$, and $\epsilon(t)$ are related, independently of any closure hypothesis, by the energy balance, obtained by integrating Eq. (1) over all wavenumbers:

$$
\dot{k}(t)=P(t)-\epsilon(t),
$$

where energy conservation by nonlinear interactions implies that

$$
\int_{0}^{\infty} d \kappa T(\kappa, t)=0 .
$$

Substituting Eqs. (9)-(11) in Eq. (15) and subtracting the steady balance $\bar{P}=\bar{\epsilon}$ gives the relation for modulated quantities

$$
-\omega \tilde{k} \sin \left(\omega t+\phi_{k}\right)=\tilde{P} \cos (\omega t)-\tilde{\epsilon} \cos \left(\omega t+\phi_{\epsilon}\right)
$$

or equivalently

$$
\begin{aligned}
& -\omega \tilde{k} \sin \phi_{k}=\tilde{P}-\tilde{\epsilon} \cos \phi_{\epsilon}, \\
& -\omega \tilde{k} \cos \phi_{k}=\tilde{\epsilon} \sin \phi_{\epsilon} .
\end{aligned}
$$

Elementary trigonometric identities give the explicit relations 


$$
\begin{aligned}
& \tilde{k}=\frac{1}{\omega} \sqrt{\left(\tilde{\epsilon} \sin \phi_{\epsilon}\right)^{2}+\left(\tilde{P}-\tilde{\epsilon} \cos \phi_{\epsilon}\right)^{2},} \\
& \tan \phi_{k}=\frac{\tilde{P}-\tilde{\epsilon} \cos \phi_{\epsilon}}{\tilde{\epsilon} \sin \phi_{\epsilon}},
\end{aligned}
$$

and the equivalent relations

$$
\begin{aligned}
& \tilde{\epsilon}=\sqrt{\left(\omega \tilde{k} \cos \phi_{k}\right)^{2}+\left(\widetilde{P}+\omega \widetilde{k} \sin \phi_{k}\right)^{2}}, \\
& \tan \phi_{\epsilon}=-\frac{\omega \tilde{k} \cos \phi_{k}}{\widetilde{P}+\omega \tilde{k} \sin \phi_{k}} .
\end{aligned}
$$

Although additional assumptions are obviously required to close the problem, explicit closure hypotheses are not required to reach some simple but useful conclusions about the limits of asymptotically high and low oscillation frequencies. Linearity implies that the frequency of the perturbation at any scale of motion must be the imposed frequency $\omega$, but in the inertial range, disturbances are damped on the Kolmogorov time-scale $\left(\epsilon^{1 / 3} \kappa^{2 / 3}\right)^{-1}$; accordingly, we anticipate that if $\omega \gg \epsilon^{1 / 3} \kappa^{2 / 3}$, the perturbations must be overdamped, but that they are active and only weakly damped if $\omega \ll \epsilon^{1 / 3} \kappa^{2 / 3}$. This argument suggests that in the static limit $\omega \downarrow 0$, the turbulence follows the slow modulations at all scales of motion, so that also $\phi_{\epsilon}(\omega), \phi_{k}(\omega) \downarrow 0$. Equation (18) then gives $\widetilde{P} \approx \widetilde{\epsilon}$; Eq. (19) is not satisfied exactly, but is approximately true since $\omega \approx 0$. Assuming that for slow modulations, the relation $\epsilon(t)=C_{\epsilon} k(t)^{3 / 2} / L$ remains valid with time-independent $L$, and that the small perturbations $\tilde{k}$ and $\widetilde{\boldsymbol{\epsilon}}$ are nearly static, then $\tilde{\epsilon} / \epsilon=(3 / 2) \tilde{k} / k$. These observations suggest that in this limit, the single-point modulated quantities admit series expansions in positive powers of $\omega$ :

$$
\begin{aligned}
& \tilde{k}=\frac{2}{3} \frac{\bar{k}}{\bar{\epsilon}} \widetilde{P}+O\left(\omega^{2}\right), \\
& \tilde{\epsilon}=\widetilde{P}+O\left(\omega^{2}\right), \\
& \phi_{k}=O(\omega), \\
& \phi_{\epsilon}=O(\omega),
\end{aligned}
$$

where the powers of $\omega$ are suggested by the parity properties of Eqs. (18) and (19) under a change of sign of $\omega$. Equivalently, to lowest order, we have

$$
\widetilde{\epsilon}=\bar{\omega} \widetilde{k}, \quad \bar{\omega}=\frac{3}{2} \frac{\bar{\epsilon}}{\bar{k}},
$$

where the frequency $\bar{\omega}$ defined by this equation is the "critical" frequency discussed by Lohse. ${ }^{7}$

In the "frozen turbulence" limit $\omega \uparrow \infty$, we see that Eq. (18) is satisfied if $\phi_{k} \approx-\pi / 2$; then

$$
\tilde{k} \approx \tilde{P} / \omega .
$$

If, as the simple argument above suggests, the perturbations are overdamped throughout the inertial range, the only scales of motion at which the oscillating force can be effective are the forcing scales themselves. If so, the modulated dissipation will also take place in this range of scales, so that

$$
\begin{aligned}
\tilde{\epsilon} & =\int_{0}^{\infty} d \kappa 2 \nu \kappa^{2} \widetilde{E}(\kappa) \\
& \approx 2 \nu \kappa_{P}^{2} \int_{0}^{\infty} d \kappa \widetilde{E}(\kappa) \approx 2 \nu \kappa_{P}^{2} \widetilde{k} \approx 2 \nu \kappa_{p}^{2} \frac{\widetilde{P}}{\omega} .
\end{aligned}
$$

As in the previous limit, the asymptotic forms Eqs. (27) and (28) do not satisfy Eq. (19) exactly, suggesting that the perturbation quantities should admit series expansions in negative powers of $\omega$ :

$$
\begin{aligned}
& \tilde{k}=\frac{\tilde{P}}{\omega}+O\left(\omega^{-3}\right), \\
& \tilde{\epsilon}=2 \nu \kappa_{P}^{2} \frac{\tilde{P}}{\omega}+O\left(\omega^{-3}\right), \\
& \phi_{k}=-\frac{\pi}{2}+O\left(\omega^{-1}\right), \\
& \phi_{\epsilon}=-\frac{\pi}{2}+O\left(\omega^{-1}\right) .
\end{aligned}
$$

\section{NUMERICAL RESULTS FROM SPECTRAL CLOSURE}

In this section, we apply the EDQNM spectral closure ${ }^{11}$ to this problem. The exact formulation of the model and the numerical method is the same as in Touil et al. ${ }^{19}$ and for details we refer to that work. In this closure, nonlinear interactions among wavenumber triads of different "shapes" are considered explicitly, with a definite weighting derived perturbatively from the governing equations.

The energy spectrum was initialized by a von Kármán spectrum; however, the influence of the initial energy spectrum vanishes after a transient and the results reported are evaluated after reaching an asymptotic state. The large scale forcing is

$P(\kappa, t)=\alpha[\bar{P}+\widetilde{P} \cos (\omega t)] \exp \left(-\gamma \kappa^{2}\right), \quad \kappa \geqslant \kappa_{0}>0$,

where $\alpha$ is the normalization constant such that $\alpha \int_{\kappa_{0}}^{\infty} \exp \left(-\gamma \kappa^{2}\right)=1$, with $\widetilde{P} / \bar{P}=0.125$ and $\gamma=0.5$. The spectral resolution is approximately 20 wavenumbers per decade. The Taylor-scale Reynolds number, defined as $\operatorname{Re}_{\lambda}$ $=\sqrt{20 k^{2} /(3 \nu \epsilon)}$ is varied between 30 and 1000. The results are shown in Figs. 1-4. In Figs. 1 and 2 we also show direct numerical simulations (DNS) results by Kuczaj et al. ${ }^{9}$ In the DNS, the value of $\widetilde{P} / \bar{P}=0.2$. To obtain a collapse of the low frequency limit, the DNS results are multiplied by the ratio between the forcing amplitudes 0.125 and 0.2 . In the DNS, one single wavenumber shell is forced. The Reynolds number varies between 30 and 50. Further details on the setup of these simulations can be found in Ref. 9 . 


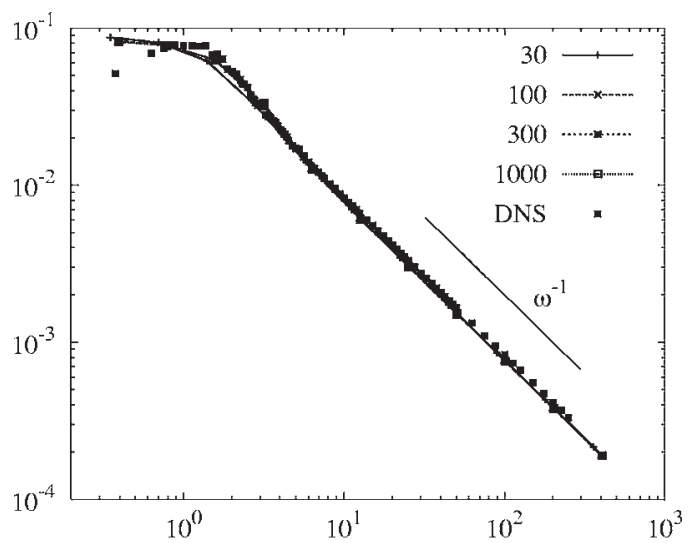

(a)

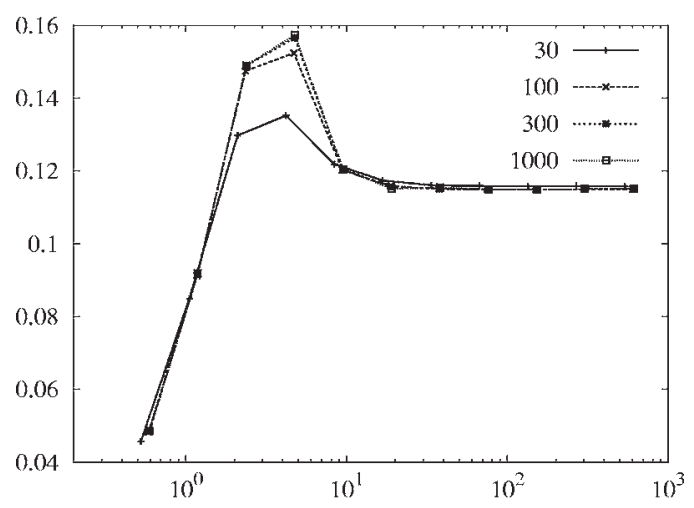

(b)

FIG. 1. Top: $\tilde{k}$ as a function of $\omega / \bar{\omega}$ for $\operatorname{Re}_{\lambda}$ varying from 30 to 1000 . Bottom: Same for $\omega \tilde{k}$.

\section{A. Modulated kinetic energy $\tilde{\boldsymbol{k}}$}

Figure 1 shows a plateau for $\tilde{k}$ at low frequencies and $\omega^{-1}$ dependence for high frequencies, as suggested by the elementary arguments leading to Eqs. (22) and (29): the static and frozen turbulence limits are well reproduced. In our calculations, no local maximum of $\tilde{k}$ is observed. In the DNS results of Kuczaj et al. ${ }^{9}$ a local maximum is present around the turbulent frequency $\bar{\omega}$ defined in Eq. (26), but in the shell model study by von der Heydt et al. ${ }^{20}$ this maximum was absent. The existence and explanation of this maximum remain open questions. However, all of the available data exhibit a clear response maximum of the compensated quantity $\omega \tilde{k}$ near $\bar{\omega}$. This maximum is also prominent in the EDQNM results shown in Fig. 1. We leave the question of whether a response maximum of $\tilde{k}$ itself is or is not consistent with closure unanswered for now. Conceivably, the answer is not universal, but may depend on the forcing scheme. The Reynolds number, or viscosity, does not seem to play an important role for $\tilde{k}$ : for moderate and high Reynolds numbers, all the data collapse on a single curve.

\section{B. Modulated dissipation $\tilde{\boldsymbol{\epsilon}}$}

Figure 2 shows that $\tilde{\boldsymbol{\epsilon}}$ also displays a plateau in the static limit, as predicted by Eq. (23). Like the compensated quantity $\omega \tilde{k}$, the compensated data $\omega \tilde{\epsilon}$ show a response maximum

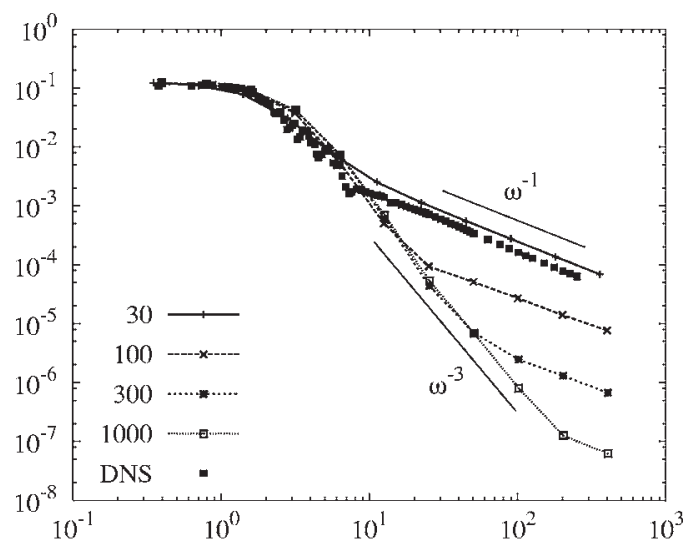

(a)

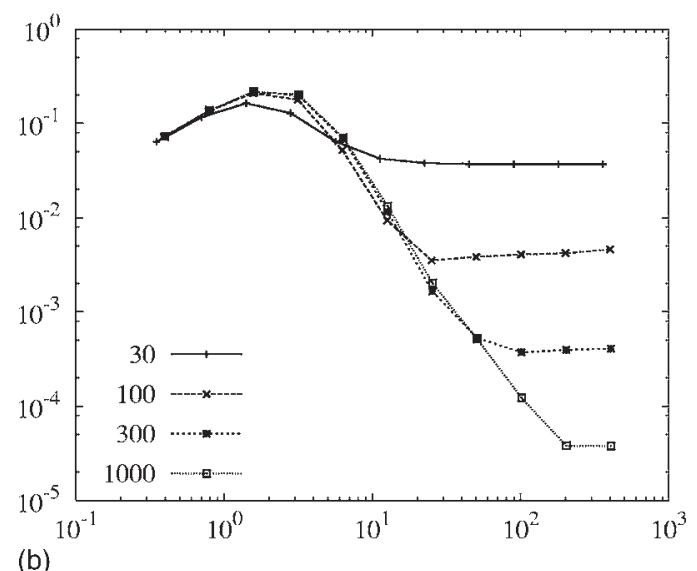

FIG. 2. Top: $\tilde{\boldsymbol{\epsilon}}$ as a function of $\omega / \bar{\omega}$ for $\operatorname{Re}_{\lambda}$ varying from 30 to 1000 . Bottom: Same for $\omega \tilde{\epsilon}$.

approximately near $\bar{\omega}$. Beyond this frequency, $\tilde{\boldsymbol{\epsilon}}$ decreases sharply; at high Reynolds number, $\tilde{\epsilon} \sim \omega^{-3}$. But at even higher frequencies, the $\omega^{-1}$ frequency dependence predicted in Eq. (30) is observed. The overall agreement with DNS is good. It is interesting to note that the high frequency $\omega^{-1}$ range depends on the Reynolds number, and is indeed proportional to the viscosity, as suggested in Eq. (30).

What remains to be explained is the fast drop of $\tilde{\epsilon}$ at intermediate frequencies. Intuitively, it can be explained as follows. At low frequencies the energy cascade can follow the modulation. At high frequencies the cascade filters the modulated energy flux, since the turbulent frequency is lower than the modulated frequency. The fast drop corresponds to the rate at which the energy cascade filters the modulated energy flux. Insights into this process have important physical consequences as they clarify how small scales are influenced by large scale forcing.

\section{Phase shifts}

Phase shift data are shown in Fig. 3. The phase lags $\phi_{k}$ and $\phi_{\epsilon}$ both go to zero for small $\omega$. In this limit, everything is in phase, as suggested by Eqs. (24) and (25). At high $\omega, \phi_{k}$ and $\phi_{\epsilon}$ go to $-\pi / 2$, consistent with Eqs. (31) and (32). A slight overshoot in $\phi_{k}$ is observed around the critical frequency. At intermediate values, $\phi_{\epsilon}$ shows a large overshoot with respect to $-\pi / 2$ and a very noticeable dependence on 


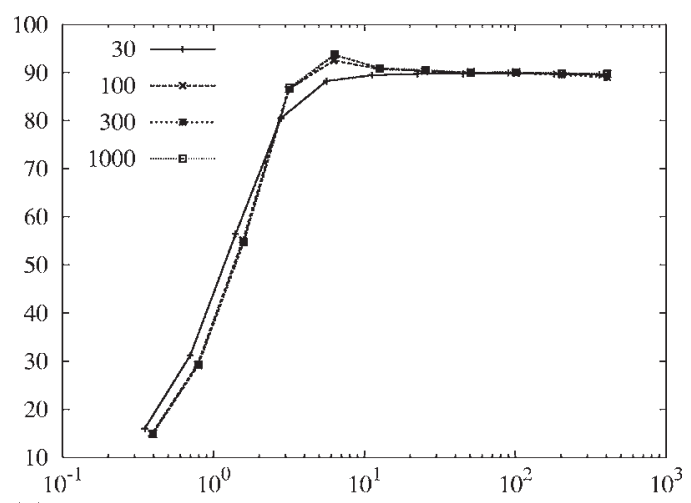

(a)

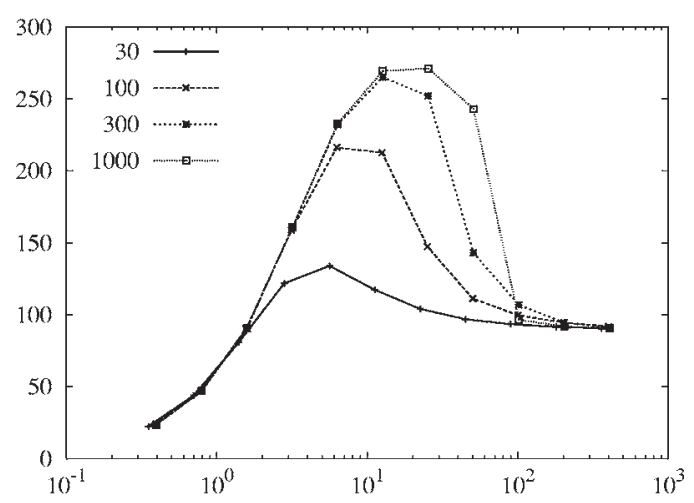

(b)

FIG. 3. Top: Phase lags $-\phi_{k}$ (in degrees) as a function of $\omega / \bar{\omega}$ for $\operatorname{Re}_{\lambda}$ varying from 30 to 1000 . Bottom: Same for phase lags $-\phi_{\epsilon}$.

the Reynolds number. This can be explained as follows: as long as the energy cascade can follow the modulation, i.e., at low frequencies, the modulated energy is transferred to the dissipation range through the energy cascade. The finite cascade time $\mathcal{T}_{c}$ introduces a phase shift between $\tilde{k}$ and $\tilde{\epsilon}$ proportional to $\omega \mathcal{I}_{c}$. This is illustrated in Fig. 4. At low frequencies, $\phi_{\epsilon}-\phi_{k}$ is a linear function of $\omega$, which permits determining the cascade time. The collapse of the curves for $\operatorname{Re}_{\lambda} \geqslant 100$ indicates that at $\operatorname{Re}_{\lambda}=100$, the asymptotic value of the cascade time is reached. At high frequencies, the oscillating contribution to the spectrum is confined to the produc-

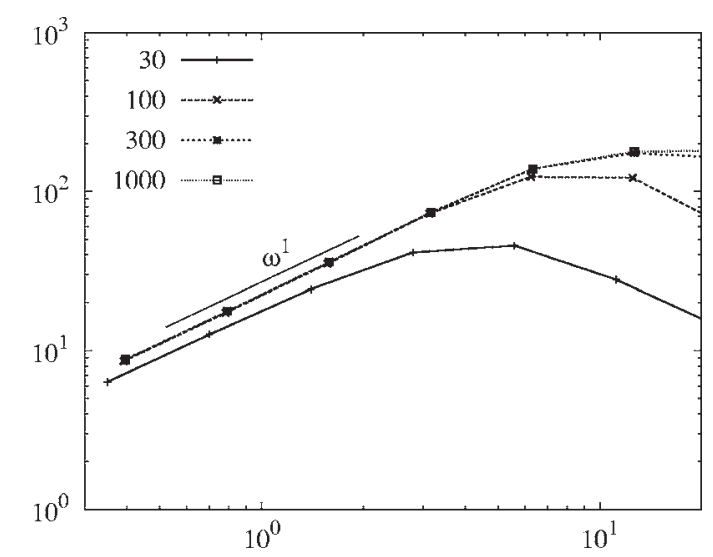

FIG. 4. $-\left(\phi_{\epsilon}-\phi_{k}\right)$ as a function of $\omega / \bar{\omega}$ for $\operatorname{Re}_{\lambda}$ varying from 30 to 1000 . tion scales. So all moments are determined by the same large scales and should roughly be in phase. Both phase shifts are $-\pi / 2$ in this limit.

\section{ANALYTICAL TREATMENT BY SPECTRAL CLOSURE}

We supplement these numerical computations with analytical results. The complexity of the EDQNM transfer integral does not permit simple direct analysis, so we will consider much simpler models that embody certain features of nonlinear turbulence dynamics, but in a way that permits analytical conclusions to be drawn relatively easily.

\section{A. General formulation}

The general closure equation is found by introducing the closure hypothesis

$$
T(\kappa, t)=\frac{\partial}{\partial \kappa} \mathcal{F}[E(\kappa, t)]
$$

in Eq. (1). Equation (34) expresses the energy transfer in terms of the energy flux $\mathcal{F}$, which is assumed to be a functional of the energy spectrum. In the problem of periodic forcing, the perturbation $\delta E(\kappa, t)$, defined by Eq. (5), satisfies

$$
\delta \dot{E}(\kappa, t)=\tilde{P}(\kappa) \cos (\omega t)-\mathcal{L}[\delta E(\kappa, t)]-2 \nu \kappa^{2} \delta E(\kappa, t),
$$

where $\mathcal{L}$ is the linear functional

$$
\mathcal{L}[\Phi(\kappa, t)]=\frac{\partial}{\partial \kappa}\left(\frac{\delta \mathcal{F}}{\delta E}\right)_{E}[\Phi(\kappa, t)]
$$

and $(\delta \mathcal{F} / \delta E)_{\bar{E}}$ denotes linearization of $\mathcal{F}$ at the steady state $\bar{E}(\kappa)$.

Separating terms proportional to $\cos (\omega t)$ and $\sin (\omega t)$, Eq. (35) can be written as

$$
\begin{aligned}
& -\omega \widetilde{G}(\kappa)=\widetilde{P}(\kappa)-\mathcal{L}[\widetilde{F}(\kappa)]-2 \nu \kappa^{2} \widetilde{F}(\kappa), \\
& -\omega \widetilde{F}(\kappa)=\mathcal{L}[\widetilde{G}(\kappa)]+2 \nu \kappa^{2} \widetilde{G}(\kappa) .
\end{aligned}
$$

In view of Eq. (36),

$$
\int_{0}^{\infty} d \kappa \mathcal{L}[\Phi(\kappa)]=0
$$

and therefore integration of Eqs. (38) and (37) recovers the single-point relations Eqs. (18) and (19).

Before beginning the analysis, we note that substituting Eqs. (37) and (38) in Eq. (13) gives

$$
\begin{aligned}
& \tilde{k} \sin \left(\phi_{k}\right)=-\frac{1}{\omega} \widetilde{P}+\frac{1}{\omega} \int_{0}^{\infty} 2 \nu \kappa^{2} \widetilde{F}(\kappa) d \kappa, \\
& \tilde{k} \cos \left(\phi_{k}\right)=-\frac{1}{\omega} \int_{0}^{\infty} 2 \nu \kappa^{2} \widetilde{G}(\kappa) d \kappa .
\end{aligned}
$$

Ignoring the viscous terms recovers $\tilde{k} \sin \phi_{k} \approx-\omega^{-1} \tilde{P}$, which is equivalent to $\widetilde{k} \approx \omega^{-1} \widetilde{P}$ and $\phi_{k} \approx-\pi / 2$, the approximations 
obtained by elementary arguments as Eqs. (29) and (31).

The corresponding substitutions in Eq. (14) yield

$$
\begin{aligned}
\tilde{\epsilon} \cos \phi_{\epsilon}= & -\frac{1}{\omega}\left[\int_{0}^{\infty} d \kappa 2 \nu \kappa^{2} \mathcal{L}[\tilde{G}(\kappa)]\right. \\
& \left.+\int_{0}^{\infty} d \kappa 4 \nu^{2} \kappa^{4} \mathcal{L}[\tilde{G}(\kappa)]\right] \\
\tilde{\epsilon} \sin \phi_{\epsilon}= & -\frac{1}{\omega}\left[\int_{0}^{\infty} d \kappa 2 \nu \kappa^{2} \tilde{P}(\kappa)-\int_{0}^{\infty} d \kappa 2 \nu \kappa^{2} \mathcal{L}[\tilde{F}(\kappa)]\right. \\
& -\int_{0}^{\infty} d \kappa 4 \nu^{2} \kappa^{4} \mathcal{L}[\tilde{F}(\kappa)]
\end{aligned}
$$

Obviously, very strong assumptions are needed to reach any conclusion about $\widetilde{\epsilon}$ and $\phi_{\epsilon}$, demonstrating that the behavior of the oscillating dissipation rate is somewhat subtle. Thus, the elementary conclusion that $\tilde{\boldsymbol{\epsilon}}$ can be approximated by taking only the first term in Eq. (42) requires arguing that the terms in $\nu \mathcal{L}$, which represent oscillatory vortex stretching, can be ignored, and that, despite the presence of $\kappa^{4}$ in the corresponding integrals, the terms in $\nu^{2}$, which represent oscillatory enstrophy destruction, can also be neglected. These assumptions are much less convincing than those underlying the elementary approximation for $\tilde{k}$. In fact, more careful analysis will reveal nontrivial features of the dynamics of the modulated dissipation. But these features can only be computed using a model; this issue will be considered in the next section.

\section{B. Simplified integral closure models}

Kraichnan $^{21}$ showed that if the correlation equation in a closure of the DIA (Direct Interaction Approximation) family is simplified by restricting attention to distant interactions only, an energy transfer model close in structure to the classical Heisenberg model is obtained. Following this observation, Rubinstein and Clark ${ }^{15}$ constructed a generalized Heisenberg model by adding asymptotically local interactions to the transfer model. The result is the energy flux closure

$$
\begin{aligned}
\mathcal{F}[E(\kappa)]= & \mathcal{C}\left\{\int_{0}^{\kappa} d \mu \mu^{2} E(\mu) \int_{\kappa}^{\infty} d p E(p) \theta(p)\right. \\
& \left.-\int_{0}^{\kappa} d \mu \mu^{4} \int_{\kappa}^{\infty} d p \frac{E(p)^{2} \theta(p)}{p^{2}}\right\},
\end{aligned}
$$

where the time argument is not explicitly written. Here and subsequently, $\mathrm{C}$ will denote some constant, but not necessarily the same constant each time it appears. This energy transfer model was supplemented ${ }^{15}$ by an evolution equation for the time-scale $\theta(\kappa)$, but the present work will use the simple algebraic closure,

$$
\theta(\kappa)=\left[\kappa^{3} E(\kappa)\right]^{-1 / 2} .
$$

The first term on the right side of Eq. (43) has the structure of the Heisenberg model: it represents energy transfer as the product of a viscosity due to modes with wavenumber larger than $\kappa$ (the second integral) times the square of a strain rate due to modes with wavenumber less than $\kappa$ (the first integral). If the model only contained this term, the energy flux would necessarily be positive, and energy would necessarily be transferred from large to small scales. The second term is a "backscatter" contribution that allows the energy flux to be negative. Then energy can also be transferred from small to large scales. The same term appears in the closely related model of Canuto and Dubovikov. ${ }^{22}$

The significance of the backscatter term, and its particular analytical form, are linked to the possibility of inviscid equipartition ensembles. Rewriting the energy transfer as

$$
\begin{aligned}
T(\kappa)= & \frac{\partial \mathcal{F}}{\partial \kappa}=\mathrm{C}\left\{\kappa^{4} \int_{\kappa}^{\infty} d p \theta(p) E(p)\left[\frac{E(p)}{p^{2}}-\frac{E(\kappa)}{\kappa^{2}}\right]\right. \\
& \left.+E(\kappa) \theta(\kappa) \int_{0}^{\kappa} d \mu \mu^{4}\left[\frac{E(\mu)}{\mu^{2}}-\frac{E(\kappa)}{\kappa^{2}}\right]\right\}
\end{aligned}
$$

shows that $T(\kappa) \equiv 0$ is consistent with $E(\kappa) \propto \kappa^{2}$. This property is lost in the classical Heisenberg model ${ }^{13}$

$$
\mathcal{F}[E(\kappa)]=\mathrm{C} \int_{0}^{\kappa} d \mu \mu^{2} E(\mu) \int_{\kappa}^{\infty} d p E(p) \theta(p),
$$

in which $T(\kappa)=0$ is only possible if $E(\kappa)=0$. Despite this conceptual drawback, the Heisenberg closure models one feature of turbulent energy transfer that will be crucial to the present analysis: the possibility of "distant" interactions between modes with disparate wavenumbers.

The linearized transfer for the generalized Heisenberg model is

$$
\begin{aligned}
\mathcal{L}[\Phi(\kappa)]= & \mathrm{C}\left\{\kappa^{2} \Phi(\kappa) \int_{\kappa}^{\infty} d p \sqrt{\frac{\bar{E}(p)}{p^{3}}}-\sqrt{\frac{\bar{E}(\kappa)}{\kappa^{3}}} \int_{0}^{\kappa} d \mu \mu^{2} \Phi(\mu)+\frac{1}{2} \kappa^{2} \bar{E}(\kappa) \int_{\kappa}^{\infty} d p \frac{\Phi(p)}{\left\{\bar{E}(p) p^{3}\right\}^{1 / 2}}\right. \\
& \left.-\frac{1}{2} \frac{\Phi(\kappa)}{\left\{\bar{E}(\kappa) \kappa^{3}\right\}^{1 / 2}} \int_{0}^{\kappa} d \mu \mu^{2} \bar{E}(\mu)-\frac{3}{2} \kappa^{4} \int_{\kappa}^{\infty} d p \frac{\bar{E}(p)^{1 / 2} \Phi(p)}{p^{7 / 2}}+\frac{3}{2} \frac{\bar{E}(\kappa)^{1 / 2} \Phi(\kappa)}{\kappa^{7 / 2}} \int_{0}^{\kappa} d \mu \mu^{4}\right\} .
\end{aligned}
$$


and the linearized transfer for the classical Heisenberg model is

$$
\begin{aligned}
\mathcal{L}[\Phi(\kappa)]= & \mathrm{C}\left\{\kappa^{2} \Phi(\kappa) \int_{\kappa}^{\infty} d p \sqrt{\frac{\bar{E}(p)}{p^{3}}}-\sqrt{\frac{\bar{E}(\kappa)}{\kappa^{3}}}\right. \\
& \times \int_{0}^{\kappa} d \mu \mu^{2} \Phi(\mu)+\frac{1}{2} \kappa^{2} \bar{E}(\kappa) \\
& \times \int_{\kappa}^{\infty} d p \frac{\Phi(p)}{\left\{\bar{E}(p) p^{3}\right\}^{1 / 2}} \\
& \left.-\frac{1}{2} \frac{\Phi(\kappa)}{\left\{\bar{E}(\kappa) \kappa^{3}\right\}^{1 / 2}} \int_{0}^{\kappa} d \mu \mu^{2} \bar{E}(\mu)\right\} .
\end{aligned}
$$

Returning to the analysis of Eqs. (37) and (38), we note that they can be decoupled to give

$$
\begin{aligned}
& {\left[\omega^{2} \mathcal{I}+\left(\mathcal{L}+2 \nu \kappa^{2} \mathcal{I}\right)^{2}\right] \tilde{F}(\kappa)=\left(\mathcal{L}+2 \nu \kappa^{2} \mathcal{I}\right) \widetilde{\mathcal{P}}(\kappa),} \\
& {\left[\omega^{2} \mathcal{I}+\left(\mathcal{L}+2 \nu \kappa^{2} \mathcal{I}\right)^{2}\right] \widetilde{G}(\kappa)=-\omega \widetilde{P}(\kappa) .}
\end{aligned}
$$

Ignoring the viscous terms in comparison to the linearized transfer gives the approximate system of equations

$$
\begin{aligned}
& \left(\omega^{2} \mathcal{I}+\mathcal{L}^{2}\right) \tilde{F}(\kappa)=\mathcal{L}[\widetilde{\mathcal{P}}(\kappa)], \\
& \left(\omega^{2} \mathcal{I}+\mathcal{L}^{2}\right) \widetilde{G}(\kappa)=-\omega \widetilde{P}(\kappa) .
\end{aligned}
$$

If $\tilde{\boldsymbol{\epsilon}}$ is computed for high oscillation frequency on the basis of the approximate Eq. (50), the result proves to be proportional to $\nu$, or of order $\mathrm{Re}^{-1}$ (with Re the Reynolds number based on the integral and lengthscale), because the frozen turbulence limit confines the oscillations to the largest scales of motion. If $\tilde{\boldsymbol{\epsilon}}$ were computed in this limit using Eq. (49) instead, the result would be to include corrections of order $\nu^{2}$, or of order $\mathrm{Re}^{-2}$. It is reasonable to ignore such corrections in the high Reynolds number limit. These considerations of high Reynolds number justify ignoring the direct viscous effects in Eq. (49) and working with the simpler system Eq. (50).

Inversion of the linear operators on the left side of Eq. (50) gives the solution for $\widetilde{F}$ and $\widetilde{G}$ in the high Reynolds number limit. However, since exact inversion is only possible numerically, we will seek asymptotic solutions for large $\omega$ using standard methods. A lowest-order approximate solution of Eqs. (37) and (38) is obtained by balancing the leading order terms in $\omega$, so that

$$
\tilde{F}(\kappa) \approx 0, \quad \tilde{G}(\kappa) \approx \frac{1}{\omega} \widetilde{P}(\kappa) .
$$

Since this result ignores nonlinearity, it might be called "rapid distortion theory" for this problem.

A formal solution of Eqs. (37) and (38) can be constructed in powers of $\omega^{-1}$ by perturbing about the leading order solution Eq. (51); taking only the correction terms of the next order gives

$$
\begin{aligned}
& \tilde{F}(\kappa)=\omega^{-2} \mathcal{L}[\widetilde{P}(\kappa)], \\
& \widetilde{G}(\kappa)=-\omega^{-1} \widetilde{P}(\kappa)+\omega^{-3} \mathcal{L}^{2}[\widetilde{P}(\kappa)] .
\end{aligned}
$$

This approximation can also be obtained by operator inversion in Eq. (50) by a Neumann series. The resulting series is divergent but asymptotic in $\omega$; therefore, as usual in such cases, the truncated series can provide useful information.

The corrections in Eq. (52) depend on $\mathcal{L}[\widetilde{P}(\kappa)]$. We note from Eqs. (48) and (47) that $\mathcal{L}[\widetilde{P}(\kappa)]$ consists of three types of terms: (1) terms proportional to $\widetilde{P}(\kappa)$, (2) terms proportional to $\int_{\kappa}^{\infty} \widetilde{P}(p) d p$, and (3) the term common to both models,

$$
\sqrt{\frac{\bar{E}(\kappa)}{\kappa^{3}}} \int_{0}^{\kappa} d \mu \mu^{2} \tilde{P}(\mu)
$$

Terms of types (1) and (2) both vanish for large $\kappa$ since $\widetilde{P}(\kappa)$ is nonzero only for small $\kappa$. These terms correspond to the intuitive idea that oscillatory disturbances are strictly confined to large scales. The important phenomenon is represented by Eq. (53), which shows that the oscillatory disturbance can indeed propagate to small scales through the effect of distant interactions between scales with wavenumber $\kappa$ and large production range scales. Because this term pertains to forward transfer alone, we obtain it in the Heisenberg model; the backscatter term in the generalized Heisenberg model therefore plays no role in this particular analysis.

Thus, the contribution to linearized transfer in Eq. (53) shows that the oscillatory disturbance is not confined to the region where $\widetilde{P}(\kappa)$ is nonzero, even at asymptotically large $\omega$, contrary to the conclusion suggested by elementary considerations. Instead, the oscillatory disturbance can propagate into all scales of motion. Eq. (53) provides the leading order solution in the regions where $\widetilde{P}(\kappa)$ vanishes.

Thus, for large $\kappa$,

$$
\mathcal{L}_{N L}[\widetilde{P}(\kappa)]=\sqrt{\frac{\bar{E}(\kappa)}{\kappa^{3}}} \int_{0}^{\kappa} d \mu \mu^{2} \widetilde{P}(\mu) \sim \kappa_{P}^{2} \widetilde{P} \bar{\epsilon}^{1 / 3} \kappa^{-7 / 3},
$$

$$
\begin{aligned}
\mathcal{L}_{\mathrm{NL}}^{2}[\widetilde{P}(\kappa)] & \sim \sqrt{\frac{\bar{E}(\kappa)}{\kappa^{3}}} \int_{0}^{\kappa} d \mu \kappa_{P}^{2} \widetilde{P} \bar{\epsilon}^{1 / 3} \mu^{-7 / 3} \\
& \sim \kappa_{P}^{2} \widetilde{P} \bar{\epsilon}^{-2 / 3} \kappa^{-5 / 3} .
\end{aligned}
$$

Adding these nonlocal contributions to the leading order solution Eq. (51), the approximation Eq. (52) takes the form

$$
\begin{aligned}
& \tilde{F}(\kappa) \sim \omega^{-2} \kappa_{P}^{2} \widetilde{P} \bar{\epsilon}^{1 / 3} \kappa^{-7 / 3}, \\
& \widetilde{G}(\kappa) \sim \omega^{-1} \widetilde{P}(\kappa)+\omega^{-3} \kappa_{P}^{2} \widetilde{P} \bar{\epsilon}^{2 / 3} \kappa^{-5 / 3} .
\end{aligned}
$$

Applying the operator $\mathcal{L}_{\mathrm{NL}}$ to both sides of Eq. (55) shows that $\mathcal{L}_{\mathrm{NL}}^{3}[\widetilde{P}(\kappa)] \sim \kappa^{-10 / 3+1}$ and that for the general power of order $p, \mathcal{L}_{\mathrm{NL}}^{p}[\widetilde{P}(\kappa)] \sim \kappa^{-10 / 3+p}$. It follows that the higher-order 
terms in the series expansions for $\widetilde{F}(\kappa)$ and $\widetilde{G}(\kappa)$ will eventually contain positive powers of $\kappa$, indicating the divergence of the series noted earlier.

Assuming that scaling ranges for $\tilde{F}$ and $\widetilde{G}$ both begin at a scale of the order of $\kappa_{P}$, we will have

$$
\begin{aligned}
& \tilde{k} \cos \left(\phi_{k}\right)=\int_{0}^{\infty} d \kappa \tilde{F}(\kappa) \sim \omega^{-2} \bar{\epsilon}^{1 / 3} \kappa_{P}^{2 / 3} \tilde{P}, \\
& \tilde{k} \sin \left(\phi_{k}\right)=\int_{0}^{\infty} d \kappa \tilde{G}(\kappa) \sim-\omega^{-1} \tilde{P}+\omega^{-3} \bar{\epsilon}^{2 / 3} \kappa_{P}^{4 / 3} \tilde{P} .
\end{aligned}
$$

If $\omega$ is large, the terms of order larger than $\omega^{-1}$ can be ignored, and we again return to the elementary estimate $\tilde{k}$ $\approx \widetilde{P} / \omega$ and $\phi_{k} \approx-\pi / 2$ with corrections depending on $\bar{\epsilon}^{1 / 3} \kappa_{P}^{2 / 3} / \omega$.

The situation is quite different for the modulated dissipation rate, for which

$$
\begin{aligned}
\tilde{\epsilon} \cos \left(\phi_{\epsilon}\right) & =\int_{0}^{\infty} d \kappa 2 \nu \kappa^{2} \widetilde{F}(\kappa) \sim \omega^{-2} \kappa_{P}^{2} \widetilde{P} \bar{\epsilon}^{1 / 3} \nu \kappa_{d}^{2 / 3} \\
& =\omega^{-2} \kappa_{P}^{2} \widetilde{P} \bar{\epsilon}^{1 / 2} \nu^{1 / 2}, \\
\tilde{\epsilon} \sin \left(\phi_{\epsilon}\right) & =\int_{0}^{\infty} d \kappa 2 \nu \kappa^{2} \widetilde{G}(\kappa) \sim \omega^{-1} 2 \nu \kappa_{P}^{2} \widetilde{P}+\omega^{-3} \kappa_{P}^{2} \widetilde{P} \bar{\epsilon},
\end{aligned}
$$

where $\kappa_{d}=\left(\epsilon / \nu^{3}\right)^{1 / 4}$ is the Kolmogorov scale. Evidently, there is a competition between the limits $\omega \rightarrow \infty$ and $\nu$ $\sim \operatorname{Re}^{-1} \rightarrow 0$. The limit $\omega \rightarrow \infty$ at fixed Re will indeed recover the elementary result $\widetilde{\boldsymbol{\epsilon}} \sim \omega^{-1}$, but at fixed large $\omega$, the limit $\mathrm{Re} \rightarrow \infty$ gives instead $\widetilde{\boldsymbol{\epsilon}} \sim \omega^{-3} \kappa_{P}^{2} \widetilde{P} \bar{\epsilon}$. The phase has the general approximate value $\tan \phi_{\epsilon} \approx \omega \nu^{1 / 2} \bar{\epsilon}^{-1 / 2}+\omega^{-1} \nu^{-1 / 2} \bar{\epsilon}^{1 / 2}$, indicating a complex joint dependence on $\omega$ and Re.

The competition between these limits reflects some basic physics of the oscillatory dynamics. To leading order, the oscillating part of the motion is confined to the production scales, giving the "frozen turbulence" approximation $\widetilde{E}_{0}(\kappa)$ $\propto \omega^{-1} \widetilde{P}(\kappa)$, as suggested by elementary arguments. The production range scales make a contribution to the energy that scales as $\operatorname{Re}^{0}$, and a contribution to the dissipation of order $\mathrm{Re}^{-1}$.

Distant interactions induce a correction $\widetilde{E}_{1}(\kappa)$ $\propto \omega^{-3} \bar{\epsilon}^{2 / 3} \kappa^{-5 / 3}$ with formally the same scaling as a Kolmogorov spectrum. This correction therefore makes $\mathrm{Re}^{0}$ contributions to oscillations of both the energy and the dissipation rate. In the case of the oscillating contribution to dissipation rate, for sufficiently large $\omega$, the $\omega^{-3}$ contribution is eventually dominated by the $\omega^{-1}$ contribution; the transition between these ranges occurs when $\omega^{-3} \sim \operatorname{Re}^{-1} \omega^{-1}$, so that $\omega$ $\sim \operatorname{Re}^{-1 / 2}$.

\section{Scaling analysis for $\tilde{\epsilon}$}

The analysis in the previous section shows how nonlocal interactions in the Heisenberg and generalized Heisenberg models can carry the oscillatory disturbance into the inertial range. These observations suggest a simple scaling analysis for the modulated energy flux. Assume, following the discussion in Sec. II that scales of motion for which the oscillations are overdamped, that is, scales satisfying $\bar{\theta}(\kappa)^{-1}>\omega$ do not transfer any modulated flux, but that modulated flux is transferred by scales of motion such that $\bar{\theta}(\kappa)^{-1}<\omega$. The crossover occurs at the scale $\kappa_{\omega}$ defined by $\bar{\theta}(\kappa)^{-1}=\omega$, or $\kappa_{\omega}$ $=\sqrt{\omega^{3} / \bar{\epsilon}}$. In both the Heisenberg and generalized Heisenberg models, the transfer of modulated flux is then given approximately by

$$
\begin{aligned}
\widetilde{\boldsymbol{\epsilon}} & \sim \int_{0}^{\kappa_{P}} d \mu \mu^{2} \widetilde{E}(\mu) \int_{\kappa_{\omega}}^{\infty} d p \bar{E}(p) \bar{\theta}(p) \sim \kappa_{P}^{2} \widetilde{k} \bar{\epsilon}^{1 / 3} \kappa_{\omega}^{-4 / 3} \\
& \sim \kappa_{P}^{2} \widetilde{P} \omega^{-1} \bar{\epsilon}^{1 / 3} \omega^{-2} \bar{\epsilon}^{-2 / 3} \sim \kappa_{P}^{2} \widetilde{P} \omega^{-3} \bar{\epsilon}
\end{aligned}
$$

This result is consistent with the existence of a contribution to $\widetilde{G}$ scaling as $\kappa^{-5 / 3}$ obtained more formally in Eq. (56).

The argument can be extended to the EDQNM closure as follows. Modulated kinetic energy is injected in the flow around the wavenumber $\kappa_{P}$. This energy will leave the large scales to enter the energy cascade at a rate $\epsilon^{\text {in }}\left(\kappa_{P}\right)$. Using classical reasoning, this rate can be estimated by

$$
\epsilon^{\mathrm{in}}\left(\kappa_{P}\right) \sim \frac{\tilde{k}}{\bar{\theta}\left(\kappa_{P}\right)}
$$

at high frequencies the modulated energy is

$$
\tilde{k} \sim \widetilde{P} \omega^{-1}
$$

and the time-scale can be estimated by

$$
\bar{\theta}\left(\kappa_{P}\right) \sim \bar{\epsilon}^{-1 / 3} \kappa_{P}^{-2 / 3},
$$

so that

$$
\epsilon^{\mathrm{in}}\left(\kappa_{P}\right) \sim \widetilde{P} \omega^{-1} \bar{\epsilon}^{1 / 3} \kappa_{P}^{2 / 3} .
$$

The point is now that this energy will be overdamped if it passes through the scales $\kappa_{P}<\kappa<\kappa_{\omega}$. The only way to reach the zone that can transfer the modulated flux, $\kappa>\kappa_{\omega}$, is by nonlocal energy transfer. This transfer will involve, for $\kappa_{P}$ $\ll \kappa_{\omega}$, triads with two legs of a length $\kappa_{\omega}$ and one leg equal to $\kappa_{P}$. The disparity parameter $s$ defined as

$$
s=\frac{\max (\kappa, p, q)}{\min (\kappa, p, q)}
$$

with $\kappa, p, q$ the norms of the wavevectors forming a triad, is for these triads

$$
s \approx \frac{\kappa_{\omega}}{\kappa_{P}} \sim \frac{\omega^{3 / 2}}{\kappa_{P} \bar{\epsilon}^{1 / 2}} .
$$

It was predicted by Kraichnan ${ }^{23}$ (compare also the DNS study by Zhou ${ }^{24}$ ), that the nonlocal part of the energy transfer involving triads with a disparity around $s, \epsilon^{f}(\kappa, s)$ with respect to the total energy flux $\epsilon^{f}(\kappa)$ scales as

$$
\frac{\epsilon^{f}(\kappa, s)}{\epsilon^{f}(\kappa)} \sim s^{-4 / 3} \text {. }
$$

In our case we identify the total flux of modulated energy, i.e., $\epsilon^{f}(\kappa, s)$, with $\epsilon^{\text {in }}\left(\kappa_{P}\right)$. The nonlocal flux $\epsilon^{f}(\kappa, s)$ corre- 


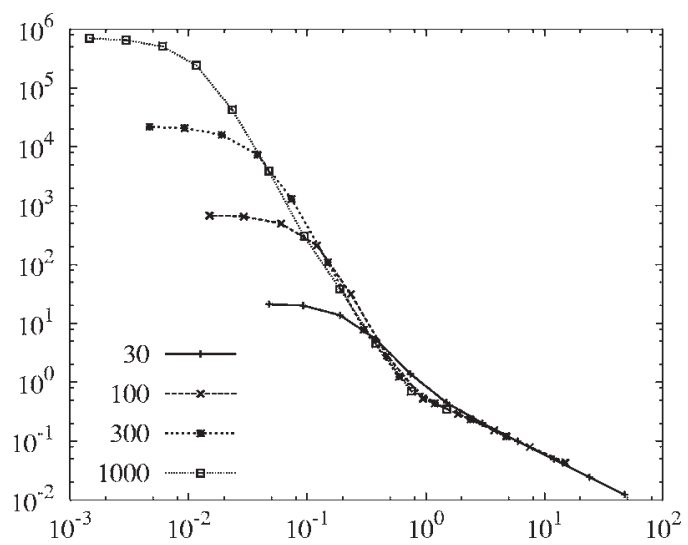

FIG. 5. $\tilde{\epsilon} /(\nu \tau)$ as a function of $\omega \tau$ for $\operatorname{Re}_{\lambda}$ varying from 30 to $1000 ; \tau$ $=\sqrt{\nu / \bar{\epsilon}}$ is the mean Kolmogorov frequency.

sponds to the modulated energy flux that manages to reach the range $\kappa>\kappa_{\omega}$ and that will eventually be dissipated, and thus is equal to $\widetilde{\epsilon}$. One finds therefore combining Eqs. (60) and (66) that the modulated dissipation for high frequencies equals

$$
\tilde{\epsilon} \sim \frac{\tilde{k}}{\theta\left(\kappa_{P}\right)} s^{-4 / 3} \sim \kappa_{P}^{2} \tilde{P} \bar{\epsilon} \omega^{-3},
$$

in agreement with Eq. (59). The inviscid nature of this correction to the modulated dissipation is in agreement with the observation in Fig. 2.

It is now possible to draw conclusions on the extension of the $\omega^{-3}$ range of $\tilde{\epsilon}$. The onset can be estimated using expression (23) for the "static" limit and (67), which yields $\omega \sim \kappa_{P}^{2 / 3} \bar{\epsilon}^{1 / 3}$, a frequency of the order of the eddy turnover frequency. The transition of the $\omega^{-3}$ range to the $\omega^{-1}$ range of $\tilde{\boldsymbol{\epsilon}}$ can be estimated by computing the intersection of expression (67) and (28). This yields a crossover of the order of the mean Kolmogorov frequency $\omega \sim \sqrt{\bar{\epsilon} / \nu}=\tau^{-1}$. In Fig. 5, the EDQNM results for $\widetilde{\epsilon}$ (Fig. 2) are replotted as a function of $\omega \tau$. The normalization of $\widetilde{\epsilon}$ by $\nu \tau$ is chosen to make the $\omega^{-1}$ range collapse.

An important distinction between the classical and generalized Heisenberg models and EDQNM is that the powerlaw scaling of Eq. (66) applies for all $s$ in the simple models, but is given by a more complex expression for EDQNM. This implies a difference in the detailed predictions when $\kappa_{\omega} / \kappa_{P}$ is of order 1 .

\section{FINITE DIMENSIONAL MODELS}

The problem of periodically forced turbulence has been investigated through properties of the single-point moments $k(t)$ and $\epsilon(t)$; complete results for these quantities have been found from various spectral closure theories. Single-point modeling attempts to circumvent spectral modeling by constructing closed equations for the single point moments themselves. It is an important theoretical question whether such equations exist, ${ }^{25}$ and indeed, much stronger assumptions are needed to close the problem at this level. In this section, we will assess how much of the dynamics is accessible to single-point modeling.
In order to permit the underlying steady state, a twoequation model for periodically forced turbulence must take the form

$$
\begin{aligned}
& \dot{k}=P-\epsilon, \\
& \dot{\epsilon}=C \frac{\epsilon}{k}(P-\epsilon),
\end{aligned}
$$

where Eq. (68) is just the energy equation previously stated as Eq. (15). For forcing at a fixed length scale, it can be shown $^{26}$ that $C=3 / 2$; the $\epsilon$ transport equation Eq. (69) then states that $L=k^{3 / 2} / \epsilon$ is constant, since Eqs. (68) and (69) imply $\dot{L} / L=(3 / 2) \dot{k} / k-\dot{\epsilon} / \epsilon=0$, the same argument that gave Eq. (22).

Equations (68) and (69) admit a steady solution in which $P(t)=\bar{P}=\epsilon(t)=\bar{\epsilon}$. We consider the perturbation about this steady state due to oscillating forcing Eq. (9); linearization about the steady state and using the value of the model constant $C=3 / 2$ gives

$$
\begin{aligned}
& \omega \tilde{k} \sin \left(\omega t+\phi_{k}\right)=\widetilde{P} \cos (\omega t)-\tilde{\epsilon} \cos \left(\omega t+\phi_{\epsilon}\right), \\
& -\omega \tilde{\epsilon} \sin \left(\omega t+\phi_{\epsilon}\right)=\bar{\omega}\left[\widetilde{P} \cos (\omega t)-\tilde{\epsilon} \cos \left(\omega t+\phi_{\epsilon}\right)\right],
\end{aligned}
$$

where Eq. (70) restates Eq. (17). Divide Eqs. (70) and (71) to obtain

$$
\frac{\sin \left(\omega t+\phi_{\epsilon}\right)}{\sin \left(\omega t+\phi_{k}\right)} \frac{\tilde{\epsilon}}{\tilde{k}}=\bar{\omega}
$$

so that

$$
\phi_{k}=\phi_{\epsilon}=\phi
$$

and

$$
\widetilde{\epsilon}=\bar{\omega} \tilde{k}
$$

The linearized equations reduce to

$$
\begin{aligned}
& \omega \tilde{k} \sin \phi=\tilde{P}-\bar{\omega} \tilde{k} \cos \phi, \\
& -\omega \cos \phi=\bar{\omega} \sin \phi .
\end{aligned}
$$

Note that this is just the general result of Eqs. (18) and (19) with the special closure hypothesis $\phi_{k}=\phi_{\epsilon}$. It follows that

$$
\tan \phi=-\frac{\omega}{\bar{\omega}}, \quad \tilde{k}=\frac{\widetilde{P}}{\bar{\omega}} \cos \phi=\frac{\tilde{P}}{\bar{\omega}} \frac{1}{\sqrt{1+(\omega / \bar{\omega})^{2}}} .
$$

The limits

$$
\begin{aligned}
& \phi \sim-\pi / 2, \quad \tilde{k} \sim \tilde{P} / \omega \text { for } \omega \rightarrow \infty, \\
& \phi \sim 0, \quad \tilde{k} \sim \tilde{P} / \bar{\omega} \text { for } \omega \rightarrow 0
\end{aligned}
$$

are consistent with the limiting results previously obtained as Eqs. (29) and (22). Whereas it is certainly expected that a two-equation model should be adequate in the static limit, it 
may be surprising that the frozen turbulence limit for $\tilde{k}$ is also predicted correctly, particularly in view of the suggestion ${ }^{5}$ that in oscillating channel flow, predicting the frozen turbulence limit requires rapid distortion theory.

Despite these successes, the two-equation model has some important limitations. First of all, the phase shifts $\phi_{k}$ and $\phi_{\epsilon}$ are equal for all $\omega$, in disagreement with Fig. 3. Second, Eq. (74) states that $\tilde{\boldsymbol{\epsilon}}$ and $\tilde{k}$ are proportional for all $\omega$. Recall that this proportionality was found in Eq. (26) as a consequence of assuming a constant length scale. Comparison of Figs. 1 and 2 shows that $\tilde{k}$ and $\tilde{\epsilon}$ are certainly not always proportional. This comparison demonstrates that the identification of the constant forcing scale $\kappa_{P}^{-1}$ with a multiple of the ratio $k^{3 / 2} / \epsilon$ cannot be made for general values of $\omega$; following the common terminology that "equilibrium" turbulence is turbulence in which all dimensional arguments are valid, we can say that periodically forced turbulence is not in equilibrium.

What is most striking is that the two-equation model cannot predict the $\nu$ dependence of $\tilde{\epsilon}$ and $\phi_{\epsilon}$, which is not a low Reynolds number effect in this case. A fundamental observation of Speziale and Bernard ${ }^{27}$ is that Reynolds number dependence in the dissipation rate dynamics is a manifestation of unbalanced vortex stretching, the absence of which underlies the classic formulation of the $\epsilon$ equation by Tennekes and Lumley. ${ }^{28}$ Even if we were satisfied with a high Reynolds number model, it should predict $\widetilde{\boldsymbol{\epsilon}} \approx 0$ for large $\omega$. We have noted that this limit is due to the filtering effect of the spectral cascade. Evidently, this effect cannot be captured at the level of a two-equation model.

Another way to understand the relation between spectral closure and the two-equation model is to note that Eq. (75) is obtained from the general closure model Eqs. (37) and (38) by making the single relaxation time approximation

$$
\mathcal{L}+2 \nu \kappa^{2} \mathcal{I} \approx \bar{\omega} \mathcal{I}
$$

before integrating over $\kappa$. This type of simplification, by which the continuum of time scales in a turbulent flow is replaced by a single dominant time scale, is a mainstay of modeling, and is often very useful; however, in the problem of periodically forced turbulence, it suppresses the nontrivial features of the finite $\omega$ dynamics.

One remedy is, as always, to argue that the model constants should be functions. If we set $C=C(\omega / \bar{\omega})$, then if $C \downarrow 0$ for $\widetilde{\omega} \uparrow \infty$, the correct behavior can be reproduced. However, this ad hoc model would have no validity apart from this very special problem and would merely amount to curve fitting.

We would like to comment briefly on the modeling of this flow with a more complex finite dimensional model with two characteristic time scales; that is, a "multiple-scale" model. ${ }^{26}$ For example, consider a three-equation model in which energy flux $f$ is distinguished from dissipation $\epsilon$. A general form for such a model that is consistent with a steady state is

$$
\begin{aligned}
& \dot{k}=P-\epsilon, \\
& \dot{f}=C_{1} \frac{f}{k}(P-f), \\
& \dot{\epsilon}=C_{2} \frac{\epsilon}{k}(f-\epsilon) .
\end{aligned}
$$

The limits $C_{1} \uparrow \infty$ and $C_{2} \uparrow \infty$ both recover the two-equation model. The primary motivation for this model is that there are now two time-scales, i.e., $k / \epsilon$ and $k / f$, instead of only one.

Linearizing as usual about the steady state

$$
\begin{aligned}
& \bar{k}+\tilde{k} \cos \left(\omega t+\phi_{k}\right), \\
& f(t)=\bar{f}+\tilde{f} \cos \left(\omega t+\phi_{f}\right), \\
& \epsilon(t)=\bar{\epsilon}+\tilde{\epsilon} \cos \left(\omega t+\phi_{\epsilon}\right) .
\end{aligned}
$$

Then,

$$
\begin{aligned}
\omega \tilde{k} \sin \left(\omega t+\phi_{k}\right)= & \tilde{P} \cos (\omega t)-\tilde{\epsilon} \cos \left(\omega t+\phi_{\epsilon}\right) \\
-\omega \tilde{f} \sin \left(\omega t+\phi_{f}\right)= & C_{1} \frac{\bar{f}}{\bar{k}}\left[\tilde{P} \cos (\omega t)-\tilde{f} \cos \left(\omega t+\phi_{f}\right)\right] \\
-\omega \tilde{\epsilon} \sin \left(\omega t+\phi_{\epsilon}\right)= & C_{2} \frac{\bar{\epsilon}}{\bar{k}}\left[\tilde{f} \cos \left(\omega t+\phi_{f}\right)\right. \\
& \left.-\tilde{\epsilon} \cos \left(\omega t+\phi_{\epsilon}\right)\right]
\end{aligned}
$$

The intervention of the new quantity $f$ in the dynamics means that $k$ and $\epsilon$ are no longer constrained to be in phase. However, prediction of the high Reynolds number result $\widetilde{\boldsymbol{\epsilon}}$ $\approx 0$ remains impossible: Equation (83) then requires $\tilde{f} \approx 0$, which is inconsistent with Eq. (82). It is not difficult to evaluate both phase lags $\phi_{f}$ and $\phi_{\epsilon}$, but even without explicit results, it is evident that Reynolds number dependence of $\phi_{\epsilon}$ remains inaccessible to this model. Although the threeequation model allows more complex phase relations and modeling of time delays in the spectral cascade, it, like the two-equation model, cannot take the Reynolds number dependence into account correctly. The addition of time scales to the dissipation rate dynamics does not solve all of the problems of two-equation modeling.

\section{CONCLUSIONS}

The influence of periodic large scale forcing on isotropic turbulence has been investigated by spectral closure theory. The asymptotic frequency dependence of the modulated energy and modulated dissipation as observed in recent simulations ${ }^{9}$ were recovered. It was pointed out that the asymptotic behavior of the modulated dissipation, which is proportional to $\omega^{-1}$, corresponds to the viscous damping of the forced wavenumbers, which is local in wavenumber space. For high and moderate Reynolds numbers, an inter- 
mediate $\omega^{-3}$ frequency dependence of the modulated dissipation was observed in the EDQNM calculations. This range characterizes the filtering properties of the energy cascade. Closures allowing for nonlocal interactions (EDQNM, classical or generalized Heisenberg) can reproduce this behavior as it corresponds to nonlocal energy transfer between the forced scales and a range of wavenumbers characterized by a crossover wavenumber $\kappa>\kappa_{\omega} \sim \sqrt{\omega^{3} / \epsilon}$. Finally, it was argued that finite dimensional models cannot correctly describe the problem of modulated turbulence.

\section{ACKNOWLEDGMENTS}

Interesting discussions with O. Cadot, F. Plaza, J.-P. Bertoglio, L. Shao, and W. van de Water are acknowledged. A. Kuczaj is thanked for kindly supplying the DNS results used in Figs. 1 and 2.

${ }^{1}$ A. J. Smits and D. H. Wood, "The response of turbulent boundary layers to sudden perturbations," Annu. Rev. Fluid Mech. 17, 321 (1985).

${ }^{2}$ S. W. Tu and B. R. Ramaprian, "Fully-developed periodic turbulent pipeflow. 1. Main experimental results and comparison with predictions," J. Fluid Mech. 137, 31 (1983).

${ }^{3}$ B. R. Ramaprian and S. W. Tu, "Fully-developed periodic turbulent pipeflow. 2. The detailed structure of the flow," J. Fluid Mech. 137, 59 (1983).

${ }^{4}$ G. J. Brereton, W. C. Reynolds, and R. Jayaraman, "Response of a turbulent boundary-layer to sinusoidal free-stream unsteadiness," J. Fluid Mech. 221, 131 (1990).

${ }^{5}$ R. R. Mankbadi and J. T. C. Liu, "Near-wall response in turbulent shear flows subjected to imposed unsteadiness," J. Fluid Mech. 235, 55 (1992).

${ }^{6}$ A. Scotti and U. Piomelli, "Numerical simulation of pulsating turbulent channel flow," Phys. Fluids 13, 1367 (2001).

${ }^{7}$ D. Lohse, "Periodically kicked turbulence," Phys. Rev. E 62, 4946 (2000).

${ }^{8}$ A. von der Heydt, S. Grossmann, and D. Lohse, "Response maxima in modulated turbulence," Phys. Rev. E 67, 046308 (2003).

${ }^{9}$ A. K. Kuczaj, B. J. Geurts, and D. Lohse, "Response maxima in time- modulated turbulence: Direct numerical simulations," Europhys. Lett. 73, 851 (2006).

${ }^{10}$ A. Staicu, C. Tipton, and W. van de Water, "Modulating turbulence," in APS Meeting Fluid Dynamics, 2003, p. 48

${ }^{11}$ S. A. Orszag, "Analytical theories of turbulence," J. Fluid Mech. 41, 363 (1970).

${ }^{12}$ C. E. Leith, "Atmospheric predictability and two-dimensional turbulence," J. Atmos. Sci. 28, 145 (1971).

${ }^{13}$ W. Heisenberg, "Zur statistischen theorie der turbulenz," Z. Phys. 124, 628 (1948)

${ }^{14}$ G. K. Batchelor, Statistical Theory of Turbulence (Cambridge University Press, Cambridge, UK, 1953).

${ }^{15}$ R. Rubinstein and T. T. Clark, "A generalized Heisenberg model for turbulent spectral dynamics," Theor. Comput. Fluid Dyn. 17, 249 (2004).

${ }^{16}$ D. Yu and S. S. Girimaji, "Direct numerical simulations of homogeneous turbulence subject to periodic shear," J. Fluid Mech. 566, 117 (2006).

${ }^{17} \mathrm{O}$. Cadot, J. H. Titon, and D. Bonn, "Observation of resonances in modulated turbulence," J. Fluid Mech. 485, 161 (2003).

${ }^{18} \mathrm{O}$. Cadot and F. Plaza, "From large scales energy injection to small scales heat dissipation in a modulated turbulent flow," in APS Meeting Fluid Dynamics, 2005, p. 59.

${ }^{19}$ H. Touil, L. Shao, and J. P. Bertoglio, "The decay of turbulence in a bounded domain," J. Turbul. 3, 1 (2002).

${ }^{20}$ A. von der Heydt, S. Grossmann, and D. Lohse, "Response maxima in modulated turbulence. II. Numerical simulations," Phys. Rev. E 68, 066302 (2003).

${ }^{21}$ R. H. Kraichnan, "An interpretation of the Yakhot-Orszag turbulence theory," Phys. Fluids 30, 2400 (1987).

${ }^{22}$ V. M. Canuto and M. S. Dubovikov, "A dynamical model for turbulence: I. General formalism,” Phys. Fluids 8, 571 (1997).

${ }^{23}$ R. H. Kraichnan, "Inertial-range transfer in a two- and a three-dimensional turbulence,” J. Fluid Mech. 47, 525 (1971).

${ }^{24}$ Y. Zhou, "Interacting scales and energy transfer in isotropic turbulence," Phys. Fluids A 5, 2511 (1993).

${ }^{25}$ S. L. Woodruff and R. Rubinstein, "Multiple-scale perturbation analysis of slowly evolving turbulence," J. Fluid Mech. 565, 95 (2006).

${ }^{26} \mathrm{R}$. Schiestel, Modélisation et Simulation des Écoulements Turbulents (Hermes, Paris, 1993)

${ }^{27}$ C. G. Speziale and P. Bernard, "The energy decay in self-preserving isotropic turbulence revisited," J. Fluid Mech. 241, 645 (1992).

${ }^{28} \mathrm{H}$. Tennekes and J. L. Lumley, A First Course in Turbulence (The MIT Press, Cambridge, MA, 1972). 